\title{
Anesthetic management of awake craniotomy with laryngeal mask airway and dexmedetomidine in risky patients
}

\author{
Yang Hoon Chung, Seulki Park, Won Ho Kim, Ik-Soo Chung, and Jeong Jin Lee \\ Department of Anesthesiology and Pain Medicine, Samsung Medical Center, Sungkyunkwan University School of Medicine, Seoul, Korea
}

Awake craniotomy is the technique of choice for the patients with lesions on eloquent cortical areas. It is still challenging for anesthesiologists, especially in cases where the patient's general condition is poor or with a relatively inexperienced surgeon. Occasionally the inability to control airway can lead to complications such as airway obstruction or hypercarbia, which can lead to brain bulging. Different anesthetic combinations have been reported for awake craniotomy. Nevertheless, there is still no consensus about the optimal regimen for awake craniotomy [1].

We experienced six patients with fronto-temporal lesion who needed awake surgery to preserve eloquent areas. Two patients had cardiac problems and one patient had chronic depression (Table 1).

All patients received scalp nerve block with $0.75 \%$ ropivacaine and $1: 200,000$ epinephrine. Three of the patients underwent "asleep-awake" technique using a laryngeal mask airway (LMA). The other patients received moderate to mild sedation. We used propofol and remifentanil infusion for sedation and two patients received dexmedetomidine infusion as an adjuvant. We did not use muscle relaxants in any cases. Mean operation time was 323 minutes and estimated blood loss was $380 \mathrm{ml}$.

One patient with severe snoring refused sedation at first and stayed fully awake until the end of cortical mapping. This patient displayed marked agitation during the awake period. Therefore, we started low dose propofol-remifentanil infusion after cortical mapping and the patient underwent intermittent apnea and hypercarbia (Patient 1). Most of the patients were maintained in a sedated state during the procedures and responded properly during cortical mapping. Two patients with LMA awakened 20-30 min after stopping infusion (Patients 2 and 3), and one (Patient 3) showed moderate hypercarbia. Other patients awakened within $10 \mathrm{~min}$ after stopping infusion. Surgeons were satisfied with patient's brain condition and response. One patient (Patient 5) with moderate sedation experienced extreme fear during the procedure. Others replied that it was endurable.

Awake craniotomy is very stressful surgery to patients. Although it is not always consistent, there is a greater possibility of extreme fear in the patients with prior anxiety history (e.g., Patient 5). Patients with medical problems, especially with cardiovascular diseases, may not tolerate the stressful environment and may lead to hemodynamic instability. In such cases, deep sedation is preferred. According to other references, most painful procedures were head pin fixation, manipulating dura, and closure after operation. Patients can easily get stressed during these procedures despite successful scalp nerve block [2]. Therefore, maintaining deep sedation or general anesthetic status during these procedures would be more helpful for patients' comfort. The most important problem of deep sedation is respiratory depression, and using airway device such as LMA may be a good choice [3]. But, it can also cause delayed awake and some residual effects with dull response or respiratory depression after extubation (e.g., Patients 2 and 3). Hypercarbia brings increased intracranial pressure in spite of normal oxygen saturation, and it can make the surgery harder. Yet, in practice, LMA re-insertion after neurologic test is very dangerous owing to head fixation posture.

Corresponding author: Jeong Jin Lee, M.D., Department of Anesthesiology and Pain Medicine, Samsung Medical Center, Sungkyunkwan University School of Medicine, 50, Ilwon-dong, Gangnam-gu, Seoul 135-710, Korea. Tel: 82-2-3410-0369, Fax: 82-2-3410-0361, E-mail: 1jj6625@gmail.com (c) This is an open-access article distributed under the terms of the Creative Commons Attribution Non-Commercial License (http:// creativecommons.org/licenses/by-nc/3.0/), which permits unrestricted non-commercial use, distribution, and reproduction in any medium, provided the original work is properly cited. 
Table 1. Summary of Intraoperative Managements and Perioperative Events

\begin{tabular}{|c|c|c|c|c|}
\hline Anesthetic management & Asleep period & Awake period & Asleep period & $\begin{array}{l}\text { Medical history/ } \\
\text { peri-operative events }\end{array}$ \\
\hline \#1. M/28 & No sedative & No sedative & P: $0.7, \mathrm{R}: 1.0$ & Severe snoring \\
\hline \multirow{5}{*}{ Sedation } & BIS: not available & BIS: not available & BIS: not available & \\
\hline & $\mathrm{PaCO}_{2}: 39.1$ & $\mathrm{PaCO}_{2}: 47.6$ & $\mathrm{PaCO}_{2}: 53.5$ & /Complained of dry \\
\hline & BP: $115-160 / 60-90$ & BP: $105-130 / 50-60$ & BP: $120-145 / 60-75$ & mouth and tickling \\
\hline & HR: $100-130$ & HR: $100-130$ & HR: $100-130$ & sense in the brain \\
\hline & $\mathrm{SpO}_{2}: 99-100$ & $\mathrm{SpO}_{2}: 97-98$ & $\mathrm{SpO}_{2}: 99-100$ & \\
\hline \multirow{7}{*}{$\begin{array}{l}\text { \#2. M/59 } \\
\quad \text { Asleep (LMA)-awake technique }\end{array}$} & $\mathrm{P}: 1.5-3.5$ & P: not used & $\mathrm{P}: 1.7-2.0$ & PCI inserted for IHD, \\
\hline & R: $1.0-2.0$ & R: no used & $\mathrm{R}: 1-1.5$ & DM, HTN \\
\hline & BIS: $30-40$ & BIS: 95-99 & BIS: $60-70$ & \\
\hline & $\mathrm{PaCO}_{2}: 33.9$ & $\mathrm{PaCO}_{2}: 49.5$ & $\mathrm{PaCO}_{2}: 49.5$ & /Dull response \\
\hline & BP: $95-145 / 50-75$ & BP: $120-155 / 60-90$ & BP: $120-145 / 65-75$ & \\
\hline & HR: $50-70$ & HR: $60-70$ & HR: $50-70$ & \\
\hline & $\mathrm{SpO}_{2}: 99-100$ & $\mathrm{SpO}_{2}: 98-99$ & $\mathrm{SpO}_{2}: 99-100$ & \\
\hline \multirow{7}{*}{$\begin{array}{l}\text { \#3. M/50 } \\
\text { Asleep (LMA)-awake technique }\end{array}$} & P: $1.5-3.5$ & P: not used & P: $0.8-1.2$ & Atrial fibrillation \\
\hline & $\mathrm{R}: 0.5-2.0$ & R: not used & $\mathrm{R}: 0.7-1.3$ & without RVR \\
\hline & BIS: $40-55$ & BIS: $95-97$ & BIS: $50-60$ & \\
\hline & $\mathrm{PaCO}_{2}: 45.4$ & $\mathrm{PaCO}_{2}: 53.4$ & $\mathrm{PaCO}_{2}: 58.4$ & /Hypercarbia \\
\hline & BP: $150-80 / 40-65$ & BP: $105-135 / 60-80$ & BP: $100-130 / 62-90$ & \\
\hline & HR: $80-100$ & HR: $90-110$ & HR: $70-100$ & \\
\hline & $\mathrm{SpO}_{2}: 99-100$ & $\mathrm{SpO}_{2}: 98-99$ & $\mathrm{SpO}_{2}: 99-100$ & \\
\hline \#4. F/44 & P: $1.0-2.0$ & P: not used & $\mathrm{P}: 1.5$ & None \\
\hline \multirow{6}{*}{ Sedation } & R: $0.5-1.5$ & R: not used & R: $1.3-1.5$ & \\
\hline & BIS: $75-80$ & BIS: $93-96$ & BIS: $69-80$ & /None \\
\hline & $\mathrm{PaCO}_{2}: 43.6$ & $\mathrm{PaCO}_{2}:$ unchecked & $\mathrm{PaCO}_{2}: 46.2$ & \\
\hline & BP: $90-120 / 40-50$ & BP: $100-135 / 40-65$ & BP: $105-120 / 45-65$ & \\
\hline & HR: $80-110$ & HR: $100-120$ & HR: $60-80$ & \\
\hline & $\mathrm{SpO}_{2}: 100$ & $\mathrm{SpO}_{2}: 100$ & $\mathrm{SpO}_{2}: 100$ & \\
\hline$\# 5 . \mathrm{F} / 61$ & P: $0.5-2.0$ & P: not used & P: $0.8-1.0$ & None \\
\hline \multirow[t]{7}{*}{ Sedation } & $\mathrm{R}: 0.5-1.8$ & R: not used & $\mathrm{R}: 1.3$ & \\
\hline & D: $0.05-0.7$ & D: not used & D: $0.1-0.5$ & /Not well sedated with \\
\hline & BIS: $77-88$ & BIS: $91-96$ & BIS: $60-80$ & high dose sedatives \\
\hline & $\mathrm{PaCO}_{2}: 41.8$ & $\mathrm{PaCO}_{2}: 30.7$ & $\mathrm{PaCO}_{2}: 37.5$ & \\
\hline & BP: $100-150 / 45-70$ & BP: $110-145 / 65 / 80$ & BP: $100-150 / 55-80$ & \\
\hline & HR: $70-90$ & HR: $50-70$ & HR: $60-80$ & \\
\hline & $\mathrm{SpO}_{2}: 100$ & $\mathrm{SpO}_{2}: 100$ & $\mathrm{SpO}_{2}: 100$ & \\
\hline$\# 6 . \mathrm{F} / 59$ & P: $0.5-4.0$ & P: not used & P: $0.5-1.2$ & Anxiety, depressive \\
\hline \multirow[t]{7}{*}{ Asleep (LMA)-awake technique } & $\mathrm{R}: 0.7-4.0$ & R: not used & $\mathrm{R}: 0.8-1.2$ & mood \\
\hline & D: 0.1 & D: not used & D: $0.02-0.1$ & \\
\hline & BIS: $30-40$ & BIS: $95-98$ & BIS: $60-65$ & /None \\
\hline & $\mathrm{PaCO}_{2}: 31.8$ & $\mathrm{PaCO}_{2}: 40.8$ & $\mathrm{PaCO}_{2}: 33.4$ & \\
\hline & BP: $105-150 / 40-70$ & BP: $130-150 / 50-70$ & BP: $100-130 / 50-60$ & \\
\hline & HR: $60-100$ & HR: $70-100$ & HR: $60-85$ & \\
\hline & $\mathrm{SpO}_{2}: 100$ & $\mathrm{SpO}_{2}: 100$ & $\mathrm{SpO}_{2}: 100$ & \\
\hline
\end{tabular}

P: propofol (target controlled infusion, $\mu \mathrm{g} / \mathrm{ml}$ ), R: remifentanil (target controlled infusion, $\mathrm{ng} / \mathrm{ml}$ ), D: dexmedetomidine $(\mu \mathrm{g} / \mathrm{kg} / \mathrm{hr}$ ).

Dexmedetomidine, which is a highly selective alpha-2 adrenoreceptor agonist, has been reported to be a useful sedative for awake craniotomy when sophisticated neurologic testing is required, because it induces less respiratory depression, less recovery agitation, and prompt response to stimuli during deep sedation [4]. In our cases, dexmedetomidine effectively decreased the possibility of respiratory depression and hypercarbia with sufficient sedation (Patient 6). Single or combination use with propofol-remifentanil is effective. But, late onset and the possibility of severe hypertension, bradycardia, or hypotension after bolus injection limit its use. We started dexmedetomidine infusion after skin incision without loading dose and slowly decreased the dose of propofol-remifentanil within the target BIS level (60 without LMA, and 40 with LMA). This combination use permitted rapid onset, and only continuous adjuvant infusion could prevent side effects related with bolus injection. Until the time of neurologic test, we could maintain sufficient sedation in spite of low dose propofol-remifentanil and it allows rapid-smooth awake (e.g., Patient 6). The response time (the interval from 
surgeon's request for awakening to LMA extubation-starting neurological test) in dexmedetomidine case was shorter (8 min, Patient 6) than LMA insertion with only propofol-remifentanil cases (20-30 min, Patient 2 and 3).

In conclusion, selecting a cooperative and tolerable patient is most important. But, in inevitable cases, the "asleep-awake" technique that uses controlled ventilation with LMA during incision period, and deep sedation with adjuvant dexmedetomidine after neurologic exam will be a safe and satisfactory procedure for both the surgeon and patient.

\section{References}

1. Conte V, Baratta P, Tomaselli P, Songa V, Magni L, Stocchetti N. Awake neurosurgery: an update. Minerva Anestesiol 2008; 74: 28992.

2. Danks RA, Rogers M, Aglio LS, Gugino LD, Black PM. Patient tolerance of craniotomy performed with the patient under local anesthesia and monitored conscious sedation. Neurosurgery 1998; 42: 28-34.

3. Sato K, Kato M. Intraoperative neurological monitoring in awake craniotomy. J Anesth 2008; 22: 493-7.

4. Rozet I. Anesthesia for functional neurosurgery: the role of dexmedetomidine. Curr Opin Anaesthesiol 2008; 21: 537-43. 